Article

\title{
Cryoconservation of Animal Genetic Resources in Europe and Two African Countries: A Gap Analysis
}

\author{
Gregoire Leroy ${ }^{1, *} \mathbb{C}$, Paul Boettcher ${ }^{1}{ }^{\oplus}$, Badi Besbes ${ }^{1}$, Coralie Danchin-Burge ${ }^{2}$, \\ Roswitha Baumung ${ }^{1}$ and Sipke J. Hiemstra ${ }^{3}$ \\ 1 Food and Agriculture Organization of the United Nations, Animal Production and Health Division, \\ Viale delle Terme de Caracalla, 00153 Rome, Italy; paul.boettcher@fao.org (P.B.); Badi.Besbes@fao.org (B.B.); \\ roswitha.baumung@fao.org (R.B.) \\ 2 Institut de l'Elevage, 149 rue de Bercy, 75595 Paris CEDEX 12, France; coralie.danchin@idele.fr \\ 3 Centre for Genetic Resources, The Netherlands (CGN), Wageningen University and Research, \\ 6708 PB Wageningen, The Netherlands; Sipkejoost.Hiemstra@wur.nl \\ * Correspondence: gregoire.leroy@fao.org
}

Received: 25 November 2019; Accepted: 10 December 2019; Published: 13 December 2019

check for updates

\begin{abstract}
Cryoconservation is one of the main strategies to conserve farm animal genetic resources, providing opportunities for genetic improvement and adaptation to changes in production environments and consumer demands. In this study, we combine livestock breed-related data from the Domesticated Animal Diversity Information System (DAD-IS) and information provided by gene banks from 15 European and 2 non-European countries on material stored for livestock breeds to analyze the gaps in cryomaterial collections according to species, countries and various breed categories. Out of the 2949 breeds registered in DAD-IS for these countries, 15.9\% have been reported to have material stored in gene banks, but only $4.3 \%$ have material sufficient to allow breed reconstitution. The proportion of breeds with stored cryomaterial was greater than $20 \%$ for ruminants and pigs, between $10 \%$ and $20 \%$ for equids, and below $10 \%$ for rabbit and avian species. According to the results of two logistic regressions, countries show significant differences in the proportion of populations collected for cryostorage, while breeds not-at-risk are more likely to have cryomaterial preserved than are other breeds. Also, a relatively larger proportion of transboundary breeds have cryomaterial in gene banks than do local breeds, likely due in part to the fact that multiple countries have the opportunity to collect this material. These results highlight the need for increased efforts in material collection for at-risk local breeds and regional coordination on cryoconservation of material from transboundary breeds.
\end{abstract}

Keywords: livestock; breed; conservation; ex situ; semen; gene bank; sustainable development goal

\section{Introduction}

According to the Domestic Animal Diversity Information System (DAD-IS) of the Food and Agriculture Organization of United Nations (FAO), 15,060 national breed populations (NBP) are currently reported by 182 countries, representing 8803 breeds and 38 species [1]. An NBP is a breed or a subpopulation of a breed found in a given country. For example, 134 countries in the world report NBPs of the Holstein cattle breed. The maintenance of livestock genetic diversity is considered as a prerequisite for the sustainability of animal production systems. Conservation strategies involve both in situ and ex situ techniques, the latter being divided between ex situ-in vivo and cryoconservation (i.e., ex situ-in vitro), which is defined as the collection and deep-freezing of semen, ova, embryos or tissues in liquid nitrogen for potential future use in breeding or regenerating animals [2]. Cryoconservation programs may have different purposes, such as breed reconstitution in 
case of extinction, support of in vivo conservation, reorientation of selection goals, research or storing of genotypes of specific interest (e.g., traits of economic importance, unique traits, cultural value). Cryopreserved material is generally stored in dedicated facilities called gene banks or cryobanks, usually hosted by research institutions, universities, government agencies or private companies. Depending on the situation, countries rely either on centralized national gene banks, or on a network of dispersed gene bank collections managed by different stakeholders [3].

The convention on Biological Diversity and the Nagoya Protocol explicitly request countries to implement complementary in situ and ex situ conservation strategies to preserve their genetic resources [4]. Also, in the framework of 17 sustainable development goals (SDG) of the United Nations, two indicators specifically address conservation of animal genetic resources (AnGR), considering the number of local breeds (see Supplementary Box 1) represented in national gene bank collections with an amount of genetic material stored that is sufficient to reconstitute the breed in case of extinction (Indicator 2.5.1.b) and the proportion of local breeds at-risk (Indicator 2.5.2) [5].

The amount of germplasm required to allow such breed reconstitution can be estimated based on the type of reproductive material available, the species, the number of donor individuals and the number of samples [2]. According to the 128 country reports prepared for the Second Report on the State of the World's Animal Genetic Resources for Food and Agriculture (SoW2; [3]), some genetic material is stored for only $15 \%$ of the NBP and only $7 \%$ of NBP have quantities of stored material that would be sufficient for population reconstitution [6]. However, according to DAD-IS, which has been used as the official repository of data for calculating Indicator 2.5.1.b since 2017, based on information provided by "national coordinators" officially nominated by countries, genetic material is stored for $4.5 \%$ of the local breeds, with only $1 \%$ of local breeds having sufficient stored material [7]. This discrepancy between the two estimates seems to be linked to under-reporting in DAD-IS.

Differences in capacity and infrastructure at country level, as well as in biotechnological development and stakeholder organization at the species level, may impact the collection of cryopreserved material. Within a given species, differences among breeds may also exist. For example, transboundary breeds found in several countries may be conserved in different numbers than rare and/or local breeds present in only a single country. The status of cryopreservation of transboundary breeds has seldom been investigated.

In the context of the EU Horizon2020 IMAGE project [8], a detailed online survey was sent directly to gene banks in European countries and was subsequently also distributed by FAO to non-European countries. The survey requested details about the breeds and the types of material stored, as well as about how the gene bank was organized and managed. Participation was voluntary and provision of partial information was allowed. By combining data from the survey and breed information from DAD-IS, the current study assesses the effectiveness of the reporting in DAD-IS and coverage of cryopreserved material according to breed categories and breed status. We aim therefore at identifying redundancies and gaps in the existing collections at national and European level, according to species, endangerment level and breed categories, and discuss recommendations and priorities for further development and rationalization of gene bank collections.

\section{Materials and Methods}

\subsection{Dataset}

Among the 61 organizations that returned the questionnaire indicating that they have germplasm collections, 42 gene banks from 15 European countries (Austria, Belgium, France, Germany, Hungary, Iceland, Italy, Latvia, Poland, Slovenia, Spain, Sweden, The Netherlands, United Kingdom, Ukraine) and 2 non-European countries (Kenya, Tunisia) provided information about their cryopreserved material. In most countries, data were provided by a single gene bank, with the significant exception of Spain, where 22 (regional) gene banks provided answers. We restricted the data analysis to 11 livestock species (excluding fish, wild species and companion animals). The dataset included the information 
about 667 NBP representing 373 local breeds and 226 transboundary breeds) (see Supplementary Box 1 for definitions). For each breed, the survey requested the number of donor animals and samples for the following types of reproductive biological material: semen, embryos, oocytes, ovarian tissue and somatic cells (males and females). The material stored is mostly semen (94.9\% of NBP with material reported) followed by embryos ( $8.2 \%$ NBP with material reported). Less than $1 \%$ of the NBP have other types of material stored.

For preliminary comparative analysis, we extracted the numbers of NBP with cryopreserved material for the 17 countries from three different datasets, namely: (i) the country reports for the SoW2 [2]; (ii) information on cryopreserved materials per NBP in DAD-IS system; and (iii) the responses to the IMAGE survey. Note some that national coordinators, which were the data providers for DAD-IS and SoW2 country reports, were also involved in the IMAGE project. Then, the list of all NBP from the corresponding species and countries were extracted from DAD-IS, for a total of 3949 NBP (2377 local and 572 transboundary breeds) and merged with the results of the IMAGE survey to identify the NBP with cryoconserved material. We created a second dataset for comparison of local versus transboundary breeds. This was accomplished by merging together (i.e., across countries) the information from NBPs representing the same regional or international transboundary breed. For example, we combined data from all NBPs of Holstein cattle across the 17 countries to form a single "breed".

In this study, the methodology chosen (and in SDG Indicator 2.5.1b) to define "sufficient" quantities of semen doses and donors for breed reconstitution is based on a $150 \%$ breed replacement plan [9]. This approach aims to preserve 1.5 times the amount of germplasm necessary to obtain, through backcrossing, 25 males and 25 females with $94 \%$ of the genetics of the conserved breed, assuming average rates of reproductive efficiency [2]. A breed was considered to have sufficient (cryopreserved) material if the number of donors was larger than 25 (in at least one of the reporting gene banks), and the number of semen samples (summed over all gene banks reporting a given breed) was larger than 250 for rabbits, 500 for avian species, 5000 for pigs (corresponding approximately to 1000 doses) and 1200 for ruminants and equids [2]. Quantities vary by species because they differ in number of offspring, length of reproductive cycles and conception rates, which influence the time and efficiency with which populations can be reconstituted.

To perform our analysis, we created different categories. The first category, called the cryoconservation status, had three groups: (i) populations (NBP or breeds depending on the dataset) with no material reported, i.e., breed reported in DAD-IS but with no material reported in the survey (no material reported); (ii) populations with some material stored, but in quantities insufficient to allow restoration of the population (material); and (iii) populations with sufficient material stored for breed reconstitution (sufficient material). Since most of the material stored was semen and no information was given about the genetic background of the donors of the stored embryos, which is needed to assess sufficiency for breed reconstruction, [2], we used only the information about semen to perform our classification. We established a second category of groups to differentiate NBP and breeds according to their endangerment status, using the SDG 2.5.2 classification: "unknown risk", "not at risk" and "at risk" (Supplementary Box 1, FAO, 2012); To evaluate differences among species, we classified NBP and breeds as either cattle, sheep, goat, pig, ass, horse, rabbit, chicken, and other avian species. Finally, we also considered the geographical status of NBP and breeds, distinguishing local, regional transboundary and international transboundary NBP (see Supplementary Box 1).

\subsection{Regression Model}

To investigate the relationship between the different categories on the cryoconservation status of NBP and breeds, we performed an ordered logistic regression, using the POLR function in the R statistical software. We used the cryoconservation status (i.e., no material reported/material/sufficient material) as a dependant variable. We analyzed two models. In model 1, cryoconservation status of NBP, species, geographical classification (i.e., local, regional transboundary, international transboundary) and country were explanatory factors. In model 2, we focused on the cryoconservation status of breeds, 
with species, geographical classification and risk status (i.e., unknown risk status, extinct, at risk, not at risk) as explanatory factors. For each explanatory factor, we estimated odds ratios and assessed the significances of differences between groups after correction for multiple testing.

\section{Results}

When comparing the three original sources of data for the 17 countries, the IMAGE survey indicated a number of NBP with material collected (657) similar to that from the SoW2 country reports (688), whereas this number was much smaller in DAD-IS (381). Among the three data sources, the number of NBP per country with material was the largest for the IMAGE survey, with the exception of Italy and France (see Figure S1). The SoW2 country reports did not provide detailed information about the NBP with stored material, so only the IMAGE survey data were used for further analysis.

As indicated previously, for the 17 countries that were represented in the IMAGE survey, response data from 3949 NBP and 2949 breeds were extracted from DAD-IS. Among these 3949 and 2949 NBP, $657(12.9 \%)$ and $596(15.9 \%)$ NBP, respectively, had cryopreserved material stored (by the IMAGE survey). As previously noted, the large majority of material reported corresponded to semen, with only $55 \mathrm{NBP}$ having stored embryos reported, mostly for cattle (32 NBP), rabbit (9 NBP) and sheep (7 NPB). Sufficient material (considering solely semen) was reported for $147 \mathrm{NBP}$ and 127 breeds ( $3.7 \%$ and $4.3 \%$, respectively). The number of NBP reported in DAD-IS varied substantially among countries, ranging from 16 (Iceland) to 710 (Germany), and so did the number of NBP with cryopreserved material, ranging from 1 (Kenya) to 107 (Spain), as well as the number of NBP with sufficient material, ranging from 0 (Hungary, Kenya, Latvia and Tunisia) to 41 (France). Not surprisingly, there was a significant relationship between the total number of NBP per country and the numbers of NBP with material or with sufficient material (Pearson correlation coefficient: $0.58, p<0.01$ ). Nevertheless, there were significant differences across countries $(p<0.001)$ in the distribution of NBP between the different cryoconservation categories (Figure 1). In particular, Austria, France, Iceland, The Netherlands and Spain reported significantly $(p<0.001)$ larger proportions of their NBP with material collected (at least $30 \%$ with material or sufficient material), when compared to other countries. For instance, the odds ratio was around 112 when comparing Spain to Kenya (see Table S1).

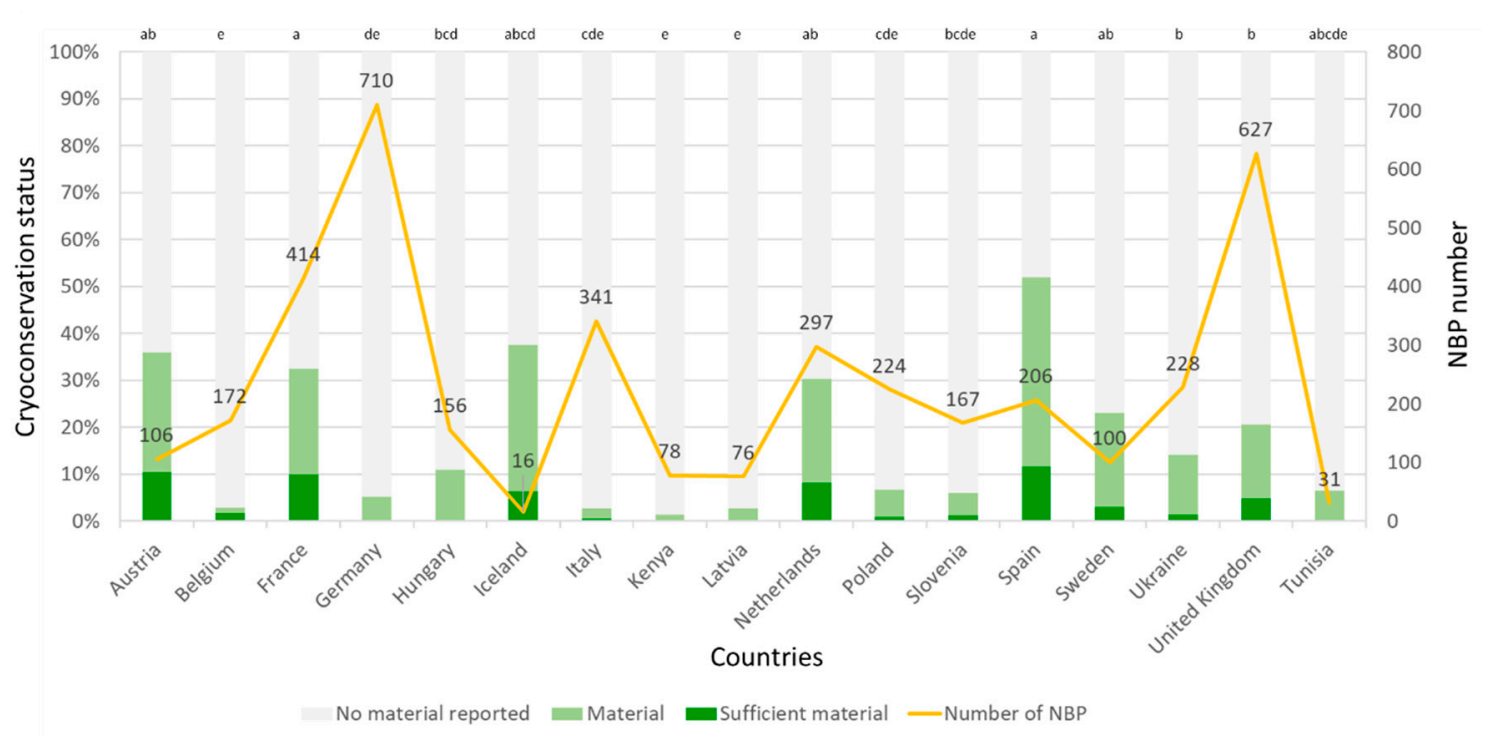

Figure 1. Cryoconservation status (in \% of national breed populations (NBP)) and number of NBP by country. Different letters indicate significant differences $(p<0.05)$ between groups after multiple correction according to the statistical model used.

Large differences were observed in terms of species coverage across countries. Among the 17 countries, 16 reported cryopreserved material for cattle, 14 for sheep, 11 for pigs, 10 for goats, 
9 for horses, 7 for chickens, and 2 each for asses, rabbits and other avians. Figure 2 illustrates the differences across species in cryoconservation status, considering either NBP or breeds. In both cases, patterns were relatively similar, with ruminants and pigs having proportions of NBP or breeds with material or sufficient material reported ranging between $20 \%$ and $30 \%$, asses and horses between $10 \%$ and $20 \%$, and rabbits, chickens and other avian species below $10 \%$. For instance, whether considering NBP or breeds, cattle showed large odds-ratios when compared to chickens (4.64 and 6.21 for NBP and breeds, respectively; $p<0.001$ ) or rabbit (9.81 and 10.85 for NBP and breeds, respectively; $p<0.001$ ) (see Tables S2 and S4). Only for cattle breeds (11.7\%) did the proportion of NBP or breeds with sufficient material to allow reconstitution exceed $10 \%$.
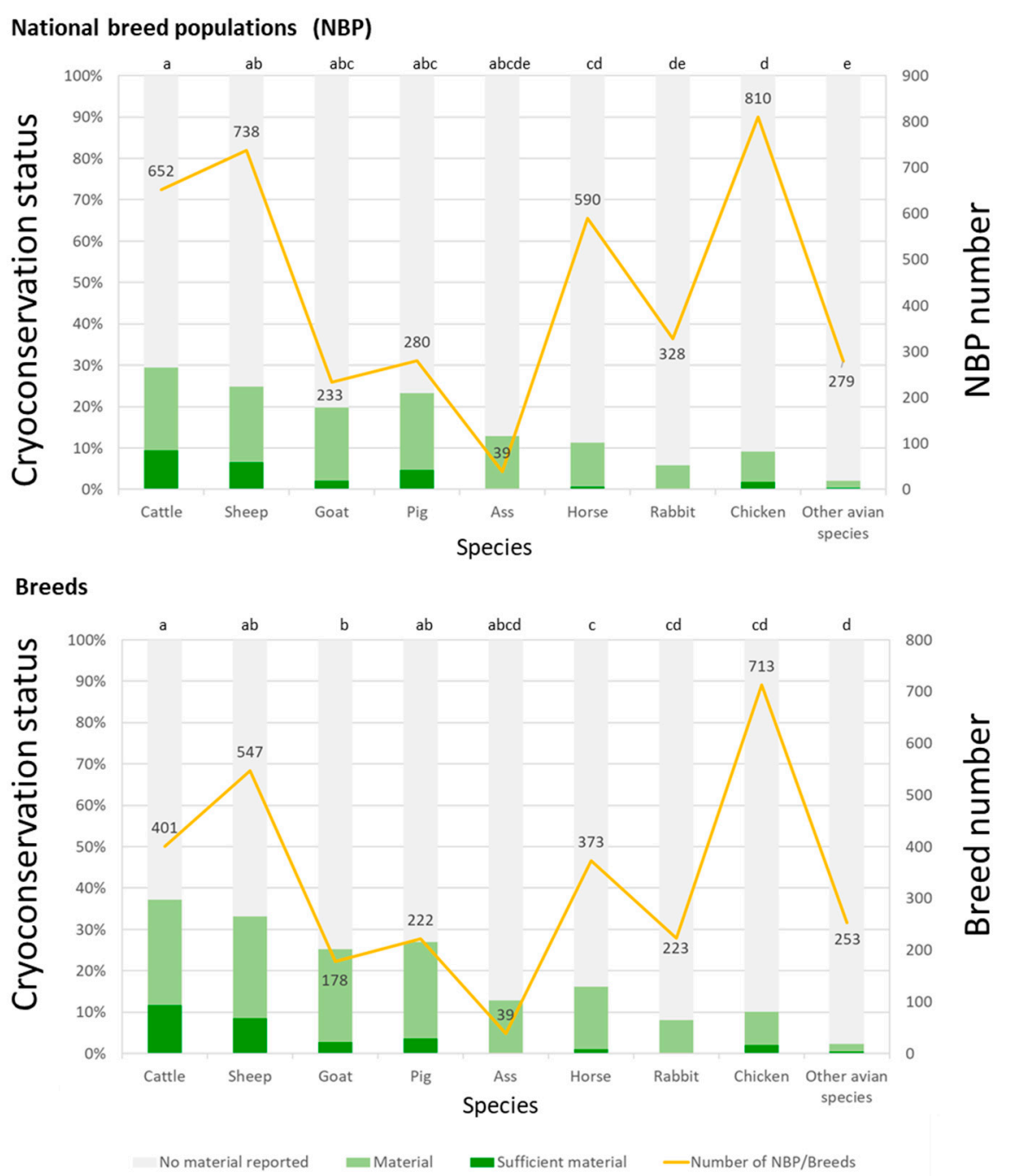

Figure 2. Cryoconservation status (in \% of NBP) and numbers of NBP and breeds according to species. Different letters indicate significant differences $(p<0.05)$ between groups after multiple correction according to the statistical model used.

Cryoconservation status according to the geographical categories is different when considering NBP or breeds (Figure 3). With NBP, we observed no significant difference at national level between international transboundary, regional transboundary or local NBP (see Table S3). In contrast, after merging the data of cryopreserved material collected in the different countries for transboundary breeds, those breeds showed a significantly higher $(p<0.001)$ proportion with material or sufficient material reported ( $43.2 \%$ and $31.6 \%$ for international and regional transboundary breeds, respectively), than local breeds (15.7\%), with odds ratios equal to 2.76 and 2.09 for the international transboundary and regional transboundary NBP, respectively, when compared to local NBP (Table S5). 

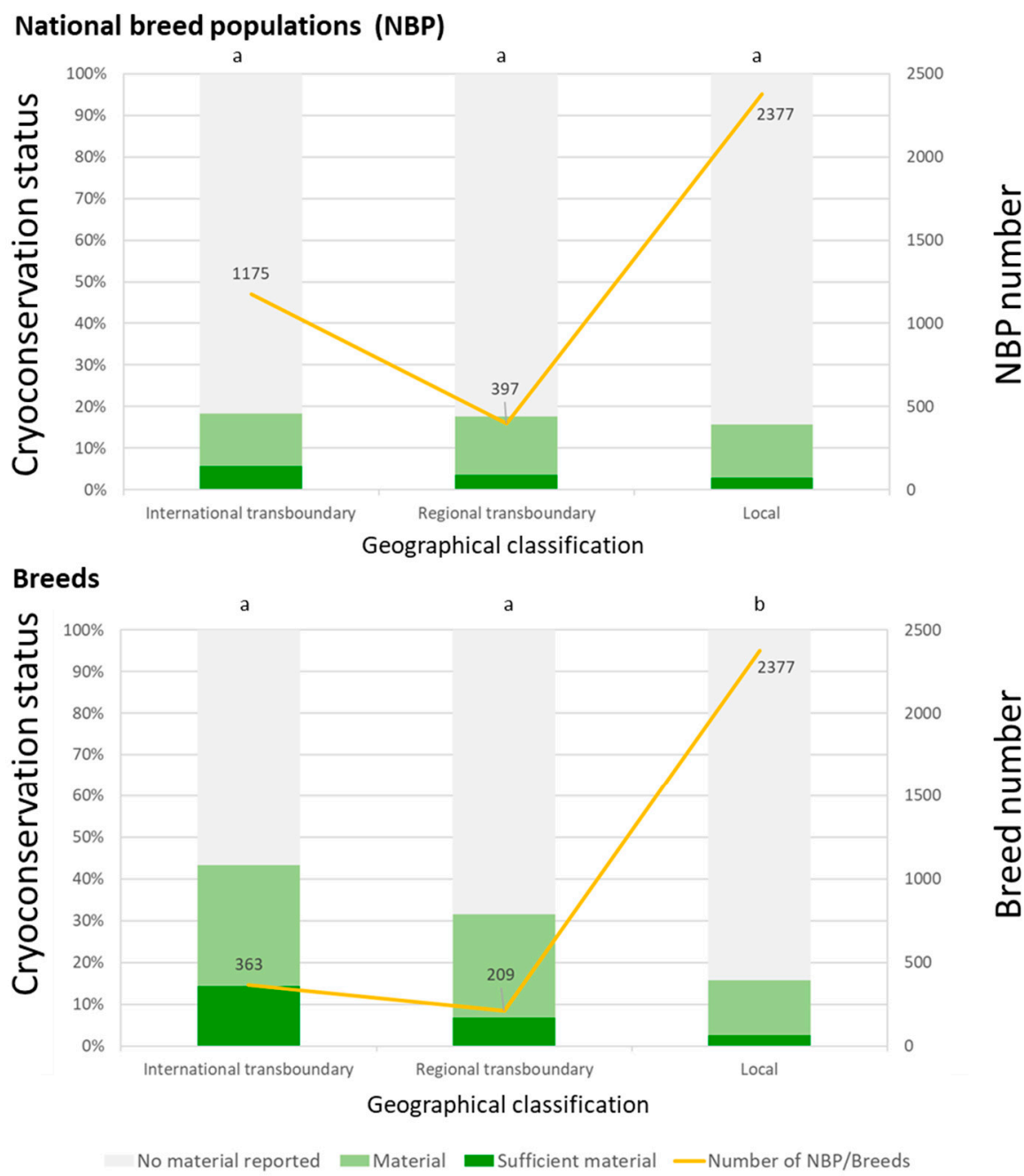

Figure 3. Cryoconservation status (in \% of NBP and breeds) and numbers of NBP and breeds according to their geographical status. Different letters indicate significant differences $(p<0.05)$ between groups after multiple correction according to the statistical model used.

When comparing the cryoconservation status of breeds in relation to their risk status (Figure 4), populations not at risk included a much larger $(p<0.001)$ percentage of breeds with material $(29.5 \%)$ or with sufficient material $(12.7 \%)$ than did breeds in other risk status categories. For instance, when comparing not at risk with extinct and unknown categories, the odds-ratios were 209 and 5.9, respectively $(p<0.001)$ (Table S6). Note that the category not at risk included a much larger proportion of regional and international transboundary breeds than did the other risk categories (Figure S2). Such breeds had more opportunity to have had cryomaterial collected (i.e., from more potential donor animals and by multiple countries). Only $1.7 \%$ and $4.7 \%$ of breeds with unknown status were reported with material or sufficient material, respectively, while a single extinct breed was reported with enough material to allow reconstitution (Topigs/Cofok Y pig line-corresponding to a commercial boar line discontinued in 2010). 


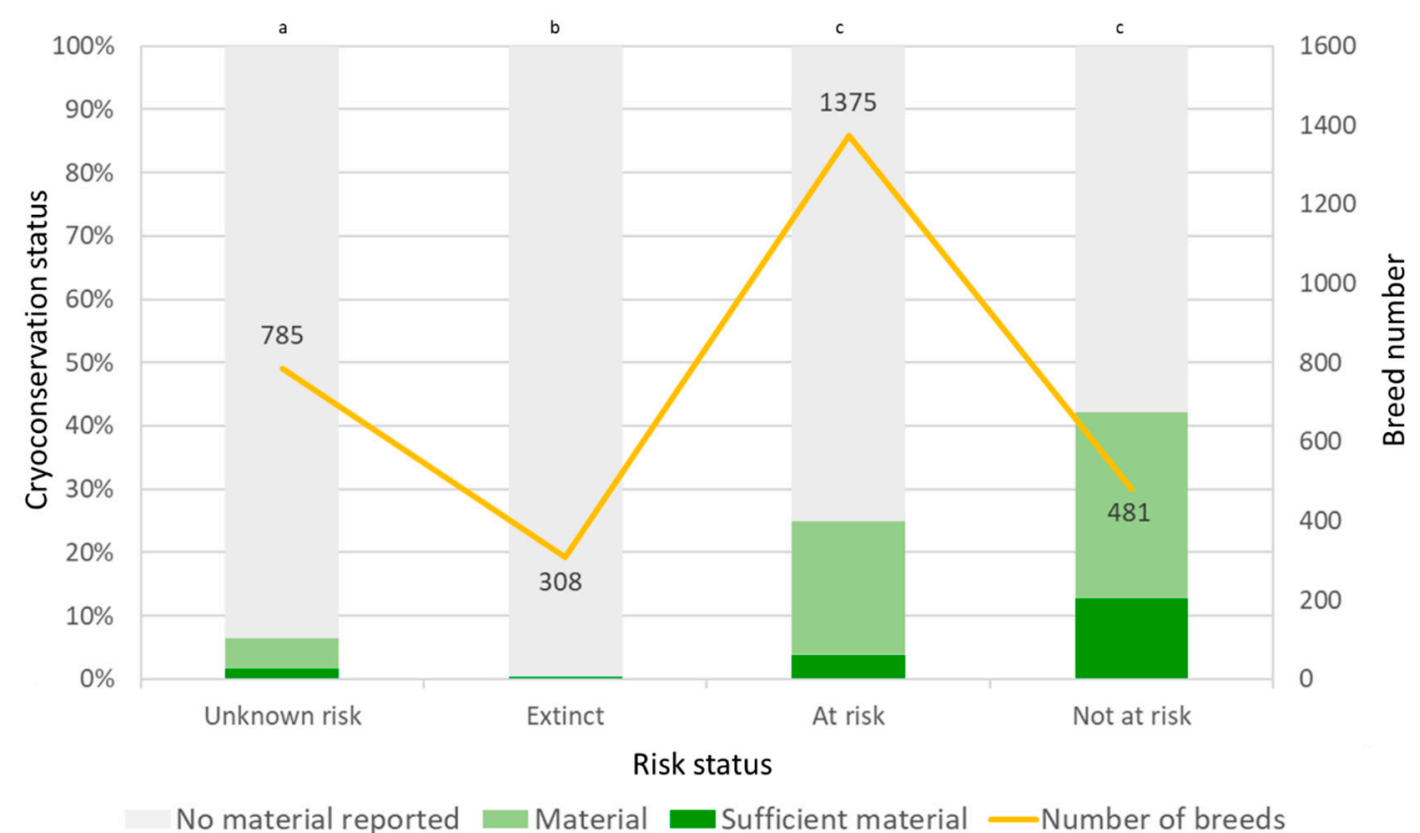

Figure 4. Cryoconservation status (in \% of breeds) and number of breeds according to their risk status. Different letters indicate significant differences $(p<0.05)$ between groups after multiple correction according to the statistical model used.

\section{Discussion}

The aim of this study was to investigate the factors that are associated with the collection of cryopreserved materials in livestock populations, using the results of a survey targeting gene banks in different European and non-European countries. In agreement with SDG Indicator 2.5.1b, only a minority of livestock breeds have cryopreserved material (mostly semen) reported to be collected and very few of these breeds have quantities of material sufficient for breed reconstitution. However, a few points to consider are as follows: (1) some breeds and NBP have sufficiently large population sizes and thus would not be priority targets for cryoconservation; (2) some breeds in the "no material reported" status may have material stored in gene banks that did not respond to the survey, thus leading to some bias in our analysis; (3) the definition of "sufficient" is based on quantities of material to reconstitute an extinct breed, which is generally the most material-intense conservation goal, whereas for some breeds and countries this may not be the conservation objective; and (4) countries are supposed to report all NBP present in the country, which typically includes both native/locally adapted breeds and exotic/imported breeds, whereas national cryopreservation programs are expected to have a strong focus on native/locally adapted breeds. All of these factors may help explain the low percentages of breeds that have material or sufficient material stored.

This survey was the first to allow a gap analysis at the population scale, taking into consideration the endangerment level of breeds, as well as the cryoconservation status of transboundary breeds, either at national level (i.e., NBP level) or by merging information on material across countries (i.e., breed level). The comparison between the survey and other datasets, i.e., the SoW2 country reports and DAD-IS data relating to cryopreserved material, demonstrated that, despite being the official structure for the computation of SDG indicators related to livestock biodiversity, DAD-IS continues to have substantial gaps in the reporting of data regarding cryopreserved material. For instance, 6 of the 17 countries that answered the IMAGE survey with gene bank data have not yet reported cryopreserved material in DAD-IS. Some bias may be present with the IMAGE survey due to under-reporting, especially in countries with no centralized system collecting information on cryopreserved material. This result highlights the need for increased exchange of information between gene banks and national coordinators, the latter of which are responsible for the data transfer into DAD-IS. Moreover, it implies the need 
for a more formal recognition of gene bank collections at national level. At the European level, the European Gene Bank Network for Animal Genetic Resources (EUGENA), governed by the European Regional Focal Point for Animal Genetic Resources (ERFP—https://www.animalgeneticresources. net) aims to strengthen the ex situ-in vitro conservation approach, complementary to the in situ conservation strategy. Monitoring of the status of animal genetic diversity at European and global levels should include both information about the amount of cryopreserved material and about the live breeding populations.

Our results show clear differences in the efforts made by the different countries to cryopreserve material of the breeds present in their territories and/or to report on these activities. For countries with a relatively low number of NBP, such as Iceland (with 16 NBP reported in DAD-IS), this effort is easier. Spain was the country with the largest proportion of NBP with cryopreserved material, in terms of both some material and with sufficient material collected, which could be somewhat related to the large number of regional gene banks in the territory and the specific policies of each bank. Spain also reported the largest proportion of NBP with material in DAD-IS, indicating that despite their large number, data are efficiently shared between gene banks and the national coordinator. The very low proportion of breeds with material reported by other countries may be related either to lack of capacity, weak policy on ex situ-in vitro conservation or lack of coordination between actors involved [3].

Artificial insemination has been widely used for decades for many species and it is thus not surprising to see frozen semen as the predominant material stored in gene banks. Embryos were less common but constituted the main material for rabbits in the two countries reporting cryopreserved material for the species (France and The Netherlands). Other materials (oocytes, ovarian tissue and somatic cells) were rarely cited, meaning that the interest or capacity of countries utilizing these materials for conservation is limited at the present time. The larger proportion of breeds with material or sufficient material stored for ruminant, pigs and equids than for avian species relates probably to the long-term existence of artificial insemination centers with dedicated animal housing and semen collection and processing facilities in many developed countries [2]. For many cattle breeds for example, semen will be commercially available and therefore easy to acquire (or receive as a donation) by the gene bank [10], meaning that specific collection and processing will not be necessary. Although substantial advances have been made in cryopreservation of avian sperm during the last fifteen years, the success of the freezing procedures remains highly variable [10]. To that extent, the relative development of reproduction technologies seems to be one of the main drivers explaining diversity among species in the extent of cryoconservation [11]. Sanitary issues may explain some of the variation within species, whereby the local breeds are kept mostly by hobby or fancy breeders (poultry mainly), which frequently have lower levels of biosecurity than do commercial farms. Financial issues also likely to play a role, favoring breeds and species for which the cost of freezing one unit of semen is small, relative to the value of a single animal. This relative cost varies from extremely low for cattle to very high for poultry. Within species, the cost of collecting a unit of material from a draft horse is much higher than the cost for a blood horse, while the economic value of the individual animals is the opposite. Our data did not allow us to account for different conservation goals on the breed level. As noted previously, breed reconstitution requires more material than other goals, such as management of diversity in situ (particularly for small populations), and some breeds may have had material collected with a less demanding objective in mind. If we had used less-demanding conservation goals to define the "sufficiency" of stored materials, then greater numbers and proportions of breeds would have achieved a sufficient cryoconservation status. However, conclusions about relative comparisons for factors such as species and geographical status would likely not have been affected (i.e., ruminants and transboundary breeds would still have had the highest proportion of breeds with sufficient materials stored).

One of the main goals of this study was to assess the extent to which a breed's presence in different countries affects its cryopreservation status. As illustrated by Figure 3, merging NBP of the same transboundary breeds together approximately doubled the proportion of populations with material or 
with sufficient material. However, it was not possible with our data to determine whether a given donor animal was represented with material in different countries' gene banks.

As underlined by de Oliveira Silva et al. [12], opportunities for increased efficiency exist in coordinating the collection and allocation of materials from transboundary breeds across countries to avoid unnecessary costs. To that extent, exchange of information on individuals stored among gene banks could help avoid the unnecessary collection and storage of material from the same or related animals in multiple countries (although some duplication may be beneficial for security purposes).

When considering the risk status of breeds, it is of interest to underline that breeds not at risk were more likely than at risk breeds to have either material or sufficient material in the gene banks surveyed. Although this may seem counterintuitive, several plausible explanations exist. First, as previously noted, breeds not at risk include a large proportion of transboundary breeds, which have opportunity to have materials collected by different countries. Second, because risk status is based on population size, breeds not at risk comprise more animals and thus, from a purely practical numeric perspective, offer more opportunities for collection of material. Also, conservation of endangered breeds is not the sole purpose of the collection of cryopreserved material, as noted by previous authors [13-15]. For any breeding program, it is relevant to cryopreserve a back-up collection as an insurance for unforeseen future circumstances. Cryopreservation actions should not wait until breeds approach extinction. Scientists and other stakeholders may benefit from the existence of an in vitro collection to support research and/or to capture specific phenotypes/alleles.

Collection of material from transboundary breeds is often easier and less expensive than collection from local breeds. Many transboundary breeds have an existing commercial value chain for their genetic material, through which semen or other material is routinely collected and thus readily available for storage in the gene bank. According to our survey, at country level, an NBP representing a transboundary breed is as likely as a local breed to have cryopreserved material collected (Figure 3). On the other hand, because there are many more local breeds than transboundary breeds, the number of collections for local breeds gene bank is much greater, indicating real efforts by countries to collect material from local AnGR.

A very small proportion of breeds with unknown risk status are cryopreserved. This result is not surprising, as (i) it may be expected that breeds that are not actively monitored are also not under an ex situ conservation program, and (ii) about half of breeds with unknown status are chicken and other avian breeds (mostly from hobby or experimental lines) which, as stated previously, are less likely as a species to have cryopreserved material. Finally, our results show almost no breeds with no living male of female animals but with material collected, which is partly explained by the fact that most of those breeds became extinct decades ago, before cryopreservation of genetic material was possible [5].

Considering either NBP or breeds, the proportions of populations with sufficient material among those with some material were nearly the same (22.3\% and $21.3 \%$, respectively). On the other hand, when considering geographical status, this proportion was much larger for transboundary breeds $(29.6 \%)$, than for local breeds $(16.4 \%)$, illustrating the potential of gaining efficiency by coordinating collection of material across countries for conservation purposes.

Finally, the thresholds chosen here (and in SDG Indicator 2.5.1b) to consider the amount of semen doses and donors sufficient for breed reconstitution can be largely discussed. Those rules consider different thresholds according to species, in relation to specific prolificacy or quality of semen. When collecting semen, ruminants produce much larger number of semen straws per ejaculate than do avian species. Such differences have likely contributed to the increased proportion of ruminants with sufficient material compared to chicken. Potential improvements in the efficiency of breed reconstitution could be achieved, for instance, by the use of sexed semen or by using genomics to estimate the relatedness among donors or to select individuals with greater proportions of the original breed during the backcross generations. However, improvements are nevertheless limited by the amount of information available on the individuals collected. Also, the amount of material necessary should also consider other factors such as the actual aim of the collection and the ownership of the 
material. If the aim of a collection is to support in situ conservation, the amount of material and the number of individuals to be collected are likely to differ from breed to breed within a species. On the other hand, breed reconstitution is probably the cryopreservation purpose requiring the largest amount of material. Therefore, it makes sense to determine a general threshold to assess the sufficient quantity of material based on the respective purpose.

\section{Conclusions}

The results of the IMAGE survey indicate collection gaps and underline several priorities in the general improvement of national and pan-European cryoconservation strategies. First, many breeds have no material in gene banks and countries, and other stakeholders should increase their efforts to initiate or expand collections, especially from local breeds at risk. This conclusion is relevant for all species, but to a lesser extent for ruminant species. This issue is relevant for European countries and for the development of the European Gene Bank Network for AnGR (EUGENA) [16], governed by the European Regional Focal Point for AnGR, but is particularly important for less developed countries, where ex situ conservation programs are often at the embryonic stage or nonexistent.

Secondly, in order to bridge the gaps existing across species, there are needs to further develop, standardize and implement reproductive technologies and cryopreservation procedures and to decrease their costs for under-represented species such as rabbits and avian species [8]. Also, even for commonly cryoconserved species such as cattle, material collection and processing procedures have typically been developed for international transboundary breeds and may thus not be optimized for less common local breeds. Finally, improvement in information exchange among gene banks, but also other stakeholders (farmers, breeding organizations, national coordinators) is required to optimize material collection and monitoring and to facilitate access and exchanges in a cost-effective manner [12,17]. Given the important gaps in the knowledge and actual collection of data related to AnGR, especially for local breeds, without greater cooperation there will be little chance to meet the goals related to SDG Target 2.5. Stakeholders worldwide should commit themselves to working together to achieve greater characterization, conservation and sustainable use of AnGR to answer the challenges posed on livestock, including by climate change.

Supplementary Materials: The following are available online at http:/www.mdpi.com/1424-2818/11/12/240/s1, Figure S1: Number of national breed populations (NBP) with cryomaterial collected according to countries and different data sources, Figure S2: Geographical status (in \% of breeds) and number of breeds according to their risk status, Table S1: Odd-ratio across countries estimated according to model 1 (national breed populations), with countries on the line above as numerators, compared to each country within the right column as denominator, Table S2: Odd-ratio across species estimated according to model 1 (national breed populations), with species on the line above as numerators, compared to each species within the right column as denominator, Table S3: Odd-ratio across geographical categories estimated according to model 1 (national breed populations), with geographical categories on the line above as numerators, compared to each geographical category within the right column as denominator, Table S4: Odd-ratio across species estimated according to model 2 (breeds), with species on the line above as numerators, compared to each species within the right column as denominator, Table S5: Odd-ratio across geographical categories estimated according to model 2 (breeds), with geographical categories on the line above as numerators, compared to each geographical category within the right column as denominator, Table S6: Odd-ratio across risk status categories estimated according to model 2 (breeds), with risk status categories on the line above as numerators, compared to each risk status category within the right column as denominator, Box 1: Glossary: populations, breeds, breed classification.

Author Contributions: Conceptualization, S.J.H., P.B. and C.D.-B.; formal analysis, G.L. and R.B.; writing—original draft preparation, G.L.; writing - review and editing, S.J.H., P.B., C.D.-B. and B.B.

Funding: This work is part of the IMAGE project which received funding from the European Union's Horizon 2020 Research and Innovation Programme under the grant agreement No. 677353.

Acknowledgments: The authors would like to thank the French government for providing for the secondment of Gregoire Leroy to the FAO.

Conflicts of Interest: The authors declare no conflict of interest. 


\section{References}

1. FAO. Status and Trends of Animal Genetic Resources-2018; Intergovernmental Technical Working Group on Animal Genetic Resources for Food and Agriculture; FAO: Rome, Italy, 2018. Available online: http: //www.fao.org/3/CA0121EN/ca0121en.pdf (accessed on 20 August 2019).

2. FAO. Cryoconservation of Animal Genetic Resources; FAO Animal Production and Health Guidelines No. 12; FAO: Rome, Italy, 2012.

3. FAO. The Second Report on the State of the World's Animal Genetic Resources for Food and Agriculture; FAO Commission on Genetic Resources for Food and Agriculture Assessments; Scherf, B.D., Pilling, D., Eds.; FAO: Rome, Italy, 2015; Available online: http://www.fao.org/3/a-i4787e/index.html (accessed on 20 August 2019).

4. CBD. The Nagoya Protocol on Access to Genetic Resources and the Fair and Equitable Sharing of Benefits Arising from their Utilization to the Convention on Biological Diversity; Convention on Biological Diversity; United Nations: Montreal, QC, Canada, 2012.

5. UNSD. Available online: https://unstats.un.org/sdgs/metadata/files/Metadata-02-05-01.pdf (accessed on 5 May 2019).

6. FAO. Status of Animal Genetic Resources-2016; Intergovernmental Technical Working Group on Animal Genetic Resources for Food and Agriculture; FAO: Rome, Italy, 2016; Available online: http://www.fao.org/3/ a-mq950e.pdf (accessed on 20 August 2019).

7. DAD-IS. Available online: http://www.fao.org/dad-is/en/ (accessed on 5 May 2019).

8. Passemard, A.; Joly, L.; Duclos, D.; Danchin-Burge, C. Inventory and Mapping of European Animal Genetic Collections. IDELE, IMAGE (Innovative Management of Animal Genetic Resources) Project Report, Paris, France. 2018. Available online: http://www.imageh2020.eu/deliverable/WP2.pdf (accessed on 20 August 2019).

9. Blackburn, H.D. Development of national animal genetic resource programs. Reprod. Fertil. Dev. 2004, 16, 27-32. [CrossRef] [PubMed]

10. Blackburn, H.D. Genebank development for the conservation of livestock genetic resources in the United States of America. Livest. Sci. 2009, 120, 196-203. [CrossRef]

11. Thélie, A.; Bailliard, A.; Seigneurin, F.; Zerjal, T.; Tixier-Boichard, M.; Blesbois, E. Chicken semen cryopreservation and use for the restoration of rare genetic resources. Poult. Sci. 2018, 98, 447-455. [CrossRef] [PubMed]

12. De Oliveira Silva, R.; Ahmadi, B.V.; Hiemstra, S.J.; Moran, D. Optimizing ex situ genetic resource collections for European livestock conservation. J. Anim. Breed. Genet. 2019, 136, 63-73. [CrossRef] [PubMed]

13. Verrier, E.; Danchin-Burge, C.; Moureaux, S.; Ollivier, L.; Tixier-Boichard, M.; Boichard, D.; Clement, F. What should be preserved: Genetic goals and collection protocols for the French National cryoank. In Proceedings of the Workshop on Cryopreservation of Animal Genetic Resources in Europe, Paris, France, 23 February 2003.

14. Danchin-Burge, C.; Hiemstra, S.J.; Blackburn, H. Ex situ conservation of Holstein-Friesian cattle: Comparing the Dutch, French, and US germplasm collections. J. Dairy Sci. 2011, 94, 4100-4108. [CrossRef] [PubMed]

15. Doekes, H.P.; Veerkamp, R.F.; Bijma, P.; Hiemstra, S.J.; Windig, J. Value of the Dutch Holstein Friesian germplasm collection to increase genetic variability and improve genetic merit. J. Dairy Sci. 2018, 101, 10022-10033. [CrossRef] [PubMed]

16. EUGENA. Available online: https://www.eugena-erfp.net/en/ (accessed on 10 September 2019).

17. Paiva, S.R.; McManus, C.; Blackburn, H. Conservation of animal genetic resources: The next decade. In Proceedings of the 10th World Congress on Genetics Applied to Livestock Production, Vancouver, BC, Canada, 17-22 August 2014.

(C) 2019 by the authors. Licensee MDPI, Basel, Switzerland. This article is an open access article distributed under the terms and conditions of the Creative Commons Attribution (CC BY) license (http://creativecommons.org/licenses/by/4.0/). 